This essay is part of IJARBM's special issue

"Contemporary Applied Business Research

in Light of Standard Academic Literature".

\title{
Leadership Seen by Neuroscience
}

\author{
Jeremie Aboiron \\ aboiron@neofaculty.org \\ Neofaculty Lab, Barcelona, Spain \\ https://doi.org/10.51137/ijarbm.2022.3.1.2
}

\begin{abstract}
In this essay, we will explain how neuroscience can be an effective tool for leaders to better manage their organizations through discovery of the human brain and how neurochemicals in the human body drive human behavior. Leadership is becoming more and more important in organizations and team management. Neuroscience can provide many insights into the various ways in which leaders can be more effective. The method used will initially be limited to a literature review. By better understanding the mechanics of neuroscience and how neurochemicals are produced in the body to affect our actions and thinking, individually, employees could use this to enable a boost in productivity. Employers can further use this biological knowledge to create a healthy culture within the organization by ensuring embedded company policies and lead in such a way that employees are happy with. Typically, this all comes back to how the organization is lead, an effective leader would know what is best for each employee and would stimulate this within the organization to balance each employee's well-being. This essay is an opening to further research on the use of neuroscience in decision-making processes, identifying stressful situations and better preparing leaders to avoid being in a critical situation.
\end{abstract}

Keywords - Leadership, Neuroscience, EEG, Behavior, Management, Corporate Culture

\section{Introduction}

Neuroscience and current technology allow us to visualize what we could not see in the past, brain activity in real time. The applications are diverse, especially in the medical field. Neuroscience is useful in a large number of areas, such as neurodegenerative diseases, language, neurological disorders following an accident, memory, etc. We will not address these issues here. Instead, we will open this topic to management, and more specifically to leadership. Today, leadership occupies an important place in the programs of business schools and universities. Companies are no longer looking for managers, but for leaders to lead their teams towards operational excellence. It is on the subject of leadership that we will focus our reflection. More precisely, we will focus on the possible applications that neuroscience can offer to the understanding and improvement of leadership. 


\section{From Cognitive Science to Leadership}

Ochsner and Lieberman (2001) defined social cognitive neuroscience as an emergent, interdisciplinary field that seeks to understand human interactions at the intersection of social, cognitive, and neural spheres of science.

Recent advances in this area offer evidence of how the human brain might support leaders in many aspects of cognition and behavior. Next, we outline three key challenges (and potential solutions) to the integration of neuroscience with management phenomena such as inspirational leadership.

These include:

- Problems in attempting to build theory that would conceptually link brain activity to leadership behavior;

- The use of expedient and effective technologies to pursue basic research that could link neuroscience to leadership;

- The lack of knowledge or technology for how to apply neurological findings to leadership development.

Henry Mintzberg (1976) addressed this challenge when he suggested that the left brain / right brain differences may be relevant to administration and leadership. As Finkelstein and Hambrick (1996) later summarized, managers with dominant left hemispheres said that approaches are largely logic and rational thinking, they can be good planners. In contrast, they suggested that managers with dominant right hemispheres, in which the focus is largely imagination, creativity, visual images and emotional response can be good managers or leaders. In particular, a neuroscientific construct known as "coherence" has facilitated the study of the origin of complex behaviors associated with leadership.

\subsection{Neuronal Coherence and Inspirational Leadership}

Regarding Waldman (2011), an important part is the leader's ability to motivate and make the commitment necessary for his employees to carry out the vision. Emotions are an important part that leaders experience and share, as well as the emotions that followers experience towards their leaders. Effective leadership involves largely regulating your own emotions, being a positive and optimistic person you transmit to your followers, thus decreasing negative feelings such as sadness, anxiety, fear that could discourage your followers. In addition, it involves understanding and the ability to influence the positive emotions of others through hope and inspiration, despite the ambiguity, setbacks or fears they might face.

Brain activities and emotions are better understood through emotional balance. Because through the equilibrio positive emotions are promoted as emotion and optimism.

For example, Heisel and Beatty (2006) found that the right frontal part of the brain was essential for effective interpersonal communication and social relationships. In addition, it has been shown that right frontal dysfunction 
results in antisocial behavior and an inability to understand relationships with other people.

\subsection{The Advancement of Neuroscience Methodologies}

Based on the work of Classen et al. (1998), who were the precursors in the field of neuroscience, we have a concrete and tested methodological framework for the use of technologies and methods applicable to the use of the electro-encephalogram. Techniques are now available to investigate brain activity that may be relevant to effective leadership behavior. These techniques vary in terms of their precision and exact capabilities (amplitude, frequency and number of waves per second), but nevertheless represent a large improvement over past techniques in their ability to detect and quantify. The data produced by qEEG can be readily used in conjunction with statistical analysis programs. In addition, qEEG is relatively inexpensive, portable, and non-invasive, and its use involves no health risk. Unlike fMRI and other methods that require people to remain immobilized in an unnatural setting (that is, in a clinical setting within a confined tube), qEEG assessment can be completed while people are comfortably seated and engaged in everyday tasks, including conversations associated with the type of vision communication task described below.

Two basic measurements are commonly used to analyze a qEEG recording:

- The amplitude, or the size of the electrical waves

- The frequency, or the number of waves per second.

Amplitude and frequency of brain waves can be assessed with regard to five bandwidths and range from low arousal (sleeping) to high arousal (extreme alertness). From lowest to highest arousal levels, these bandwidths are known as delta, theta, alpha, beta, and gamma rhythms. In the cases bellow, you'll see the brain activity in the beta rhythm because beta waves are the most prevalent in the alert brain. In addition, regarding Classen et al. (1998), beta waves are involved with affective and cognitive processes, selective attention, concentration, and anticipation. More will be said below regarding our use of beta waves and coherence in our study of the relationship between neurological functioning and inspirational leadership. 


\subsection{Illustrations}

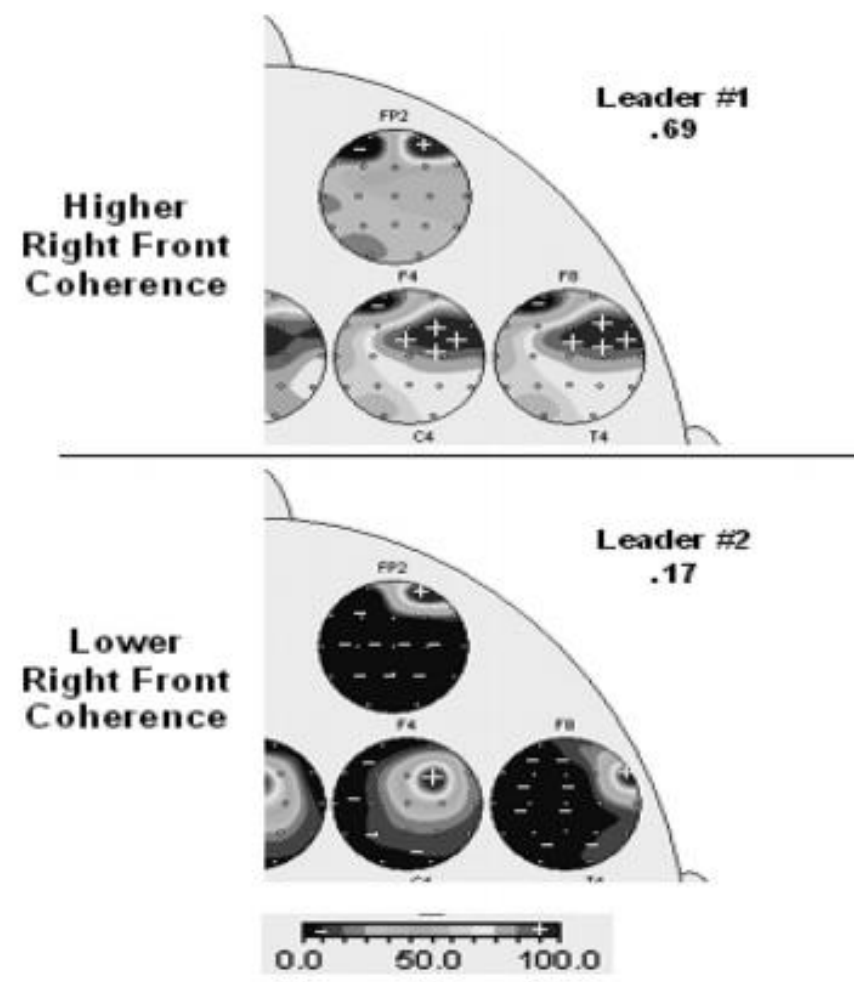

Figure 1 : Case Examples of the Right Front Coherence of Leaders, Waldman (2011)

- Dark regions with plus (+) signs represent areas with high degrees of coherence ( $75 \%$ or higher)

- While dark regions with minus (-) signs represent areas with low coherence $(25 \%$ or lower).

Leader \#1 is a 52 -year-old leader of a private non-profit, communitybased corporation that provides health and human services to Hispanic communities. Based on evaluations by followers, Leader\#1 rated very high on inspirational/charismatic leadership.

The qEEG analysis revealed high frontal right hemisphere coherence $(69 \%)$ for this individual.

Leader \#2 is a 48-year-old senior executive in an engineering/construction firm. In the housing boom of the early 2000s, leader \#2 changed employers several times while simultaneously gaining higher-level management positions. Leader \#2 also produced one of the lowest scores recorded in our research in terms of follower ratings of inspirational/charismatic leadership. Moreover, this person's brain activity presented only limited coherence $(17 \%)$ in the right frontal regions, a marked contrast to the patterns seen in leader \#1. 
Through these studies you can notice the differences that exist between the leaders and the technical help and the techniques that help to improve each of them.

\subsection{Implications for Research and Leadership Development}

We know that Day's work (2000) explores individual leaders, and more specifically the set of leadership processes that positively influence the organization.

After seeing the previous results of the studies it can be demonstrated that the neuroscience can provide information may be produced to model leadership process. Moreover, new techniques or measures may be developed to more precisely assess leadership potential. The question is now : can We Apply Neurological Findings to Leadership Development?

Through neuroscience, we can provide new techniques to achieve these objectives. These questions could shed new light on whether leaders were born versus those made, and on the effectiveness of leadership development versus natural capacities.

To this end, the objective would be to be able to assess the leadership potential through neuroscience and then use it to more directly develop the neurological wiring associated with effective leadership behaviors.

Thus, by understanding the neurological bases for inspirational behavior, we may be better positioned to develop inspirational leaders in a more realistic and systematic manner. For instance, instead of simply putting people through a one-sizefits-all leadership development program, the brain may give us some insight as to how and why specific individuals in leadership roles feel, think, and ultimately learn new behaviors.

Neurofeedback can help improve one's ability to regulate certain bodily reactions, such as responding to stress with less reactivity and intensity. As such, neurofeedback may be useful for those who want to function to the best of their capabilities. On the basis of such developments in neurofeedback technology, we may be able to directly address the neural pathways associated with inspirational leadership behaviors.

it is also important to recognize the moral and ethical implications of work directed toward linking neuroscience and leadership. The forms of leadership investigated here represent positive, constructive approaches toward influencing individuals and groups. However, we acknowledge that a darker side exists with regard to leadership in the form of manipulation and personalized vision, like House \& Howell (1992) said. It is imperative that future efforts to use neurofeedback for the purpose of leadership development be cognizant of such distinctions, and that attempts be made to promote only positive uses of such technologies.

In short, they are three key challenges in the pursuit of applications of neuroscience to leadership assessment and development. Research could now allow us to better understand the role of the brain in producing effective leadership behaviour and explore how the brain itself could be used to better develop exemplary leadership potential. This knowledge could be useful to organizations that are making significant efforts to identify and improve 
leadership skills and behaviors and I think it will be an essential tool for companies.

On the other hand, Day, Gronn and Salas (2004) assumed that leadership is a form of social capital that is linked to organizational theory, i.e. knowledge sharing, the learning organization, etc.

There are many challenges in this area, and it should always be applied ethically since it is a great responsibility to use technology with neuroscience.

\section{Neurosciences can really help us become better leaders?}

Based on Nolan's work (2013), leadership and Corporate culture are highly influential in the corporate business world. Effective leadership is the key to the development of organizations to become more productive, increase profitability and produce a better-quality workforce. As we seen before, neuroscience is an effective tool for leaders to better manage their organizations through discovery of the human brain and how neurochemicals in the human body drive human behavior. Leveraging this knowledge can enable leaders to develop a healthier corporate culture within an organization.

In the seventeenth and eighteenth Century, where science was still being revolutionized, management theories were simple ideas and usually considered impractical to be applied to organizations. A classic type management theory that naturally embedded itself showed that leaders are usually domineering, high in the hierarchy, shows little empathy for the wellbeing of the employees and highly task oriented. Leaders were generally only profit driven, and anyone that would conflict with the monetary implications of the organization were usually fired. The combination of these circumstances, and working conditions, meant that employees were unhappy with their job, and felt insecure in the workplace. From a biological perspective, these unpleasant feelings caused harmful neurochemicals in the body which effective the productivity and focus of an employee. However, leaders have the to change the culture within an organization by stimulating beneficial neurochemicals instead.

Organizational Culture can be described as the personality of an organization, not just the personality of a single employee, but rather a group of people working in the company. Organizational culture is much larger than this, it encumbers how things work in the organization, how employees communicate with their managers, how employers treat their employees and how employees interact and work with each other. An organization's productivity and effectiveness are closely related to its working culture, which can be generated by management from top-down, or self-generated amongst its employees. One thing that stands out from all this, is that the culture of an organization is something that can be manipulated which is typically driven by leaders. This means the actions of a leader within an organization is highly contributable to the cultivation of an organization. 


\subsection{Fundamentals of neuroscience and neurochemicals}

Neuroscience refers to the scientific study of the nervous system in the human body. The way in which we think, feel and behave is driven by the nervous system. By better understanding the mechanics of neuroscience and how neurochemicals are produced in the body to affect our actions and thinking, individually, employees could use this to enable a boost in productivity. By limiting the amount of harmful neurochemicals and encouraging the beneficial neurochemicals, this can lead to a better working mood and an increase in employee productivity. Employers can further use this biological knowledge to create a healthy culture within the organization by ensuring embedded company policies and lead in such a way that employees are happy with. This in turn motivates employees and encourages them to work 'the extra mile'.

Regarding to Zhe (2015) neurochemicals generally comprise of neurotransmitters and other neurotic drugs that play their specific role within the body's central nervous system. The prominent drugs would be oxytocin, serotonin, dopamine, cortisol, endorphins and so on. Each of these neurochemicals plays its own role in how we act to external stimulus. The production of these chemicals directly drives human behaviour as the nervous system is connect to the human brain. For example, when a person is facing imminent danger, the body produces high levels of cortisol, which increases a person's heart rate, blood pressure and sugar levels which in turn makes a person highly attentive and alert to one's surroundings. This gives the individual a sudden boost of energy and lowers the immune system response to prepare for a higher tolerance for pain. The body has essentially entered the fight or flight mode due to the increased presence of Cortisol.

\subsection{Effective Leadership and Healthy Organizational Culture}

Regarding Nolan (2013), leadership refers to the appointment of a person to lead a group or organization to certain direction to accomplish a set of tasks. There are many leadership styles, and each style has its own pros and cons. Over the evolution of leadership, one leadership style has not been defined as the best single contingency style, as human beings are extremely complex in nature. However, to be an effective leader, there is still a number of fundamental characteristics one must possess.

Leaders typically show qualities of being competent and ambitious. They are highly motivated and competitive to strive to be at the top of their game to rise above competitors. Leaders play a role of energising the workforce through competition. When an organization shows no competition at the marketplace, it generally lowers the job performance of employees and become complacent. Other roles of a leader is to think and plan strategically on how to grow and expand the organization through a long term vision. A vision is extremely important within an organization and must be clearly communication to ensure the entire organization is working towards common goal. This enables employees to focus and be productive, as employee who fail to find a purpose in what they do, generally have poor job performance. 
Communication is crucial to be an effective leader, by engaging his employees or team in a rational manner. Leaders have a responsibility to communicate the organizations vision in order to create a common goal for the workforce to strive for. The best leaders have open and broad minds and are attentive to listen. Cherishing employees and appreciating ideas and feedback, encourages employees to a sense of belonging. This builds a sense of trust between leaders and employees and starts to develop a healthy working culture. A happy working culture motivates employees to achieve long and short-term goals.

\subsection{The chemistry of neuroscience}

Dopamine - Regulates memory, mood, motivation, sexual, arousal, orgasm, fear and learning. Usually triggered from tasting good food or a sense of accomplishment. A negative side effect that can occur is addiction to Dopamine.

Cortisol - Temporary disables immune system and suppresses metabolism. It is typically stress induced and usually when a person is under pressure or facing fear. Cortisol is good in small doses, but longer exposure can suppress the immune system a person can become prone to disease.

Serotonin - Happiness, regulates sleep, memory, appetite, mood and body temperature. Usually produced with a sense of significance or pride. Low levels of Serotonin can cause anxiety, fear, self-pity, stress, insomnia and also depression.

Oxytocin - 'Love Hormone' which leads to mutual trust, empathy, cordiality and intimacy. It is stimulated when a person is being taken care of, or inversely when caring for others. A lack of Oxytocin leads to fear and anxiety, where as an increase leads to social bonding, faster rate of healing wounds and increased generosity. Oxytocin can be affective and contagious. It can be released by even an act of a single handshake, or even a person witnessing a handshake too.

These molecules can have an impact on our work because they influence our behavior, our state of mind, our energy, and consequently our fatigue. Dopamine is the famous pleasure molecule. The work of Gilgenkrantz (1997), and studies in positive psychology have proven that professional success comes from people with a high level of dopamine. Conversely, cortisol is the hormone that blocks our brain and activates our muscles, especially to escape danger or major stress. All this explains how hormones affect our brain, our well-being, and our ability to react to situations.

Excess levels of Dopamine in an organization can lead to addiction. If a leader sets a target and it is achieved, it can release Dopamine. A leader may become addicted to this feeling, and push the employees harder, regardless of their well-being. Similar to someone that is addicted to drugs, they would to anything in their power to get those drugs and jeopardise relationships with family and friends to do this.

Cortisol can also contribute to an organizations toxic culture if employees are emotionally unstable. A bad working environment can increase Cortisol levels which, in turn, increases hyper alertness and "desk rage" in 
employees. If an employee is continually stressed and therefor facing long term exposure to Cortisol, it can suppress the immune system and lead to disease. In extreme cases, this may lead to burnouts or even depression. The combination of these risk can lead to loss of productivity and efficiency within an organization.

\section{Conclusion}

Leaders need to cultivate a healthy corporate culture by stimulating beneficial neurochemicals in the workplace. Although this needs to be done on an individual basis, the collective stimulation of Oxytocin and Serotonin in each employee with collectively cultivate a good and healthy corporate culture. Serotonin builds leaders and employees to work more creatively and take on initiatives with more focus. The mixture of oxytocin and serotonin creates a phenomenon where leaders can delegate and empower employees to perform certain tasks. Furthermore, in these conditions, employees are not under much stress and know their leaders have a higher acceptance of failure or mistakes. With a proper mindset and attitude, an employee can carry out tasks more effectively.

Neurochemicals can be used as management tools for leaders to improve work quality within an organization. A toxic culture can be overcome by stimulating Oxytocin and Serotonin. Dopamine can be stimulated within an organization that lacks vision and motivation by creating vision and goals. To make an organization more attentive, Cortisol can used to push the workforce. Typically, this all comes back to how the organization is lead, an effective leader would know what is best for each employee and would stimulate this within the organization to balance each employee's well-being. Keeping in mind that a Leader must also ensure an emphasis still remains on the organization's profitability.

\section{$5 \quad$ References}

Alexander, S. P., Mathie, A., \& Peters, J. A. (2008). Guide to receptors and channels (GRAC). British journal of pharmacology, 153(S2), S1-S1.

Brown, S. M., \& Hariri, A. R. (2006). Neuroimaging studies of serotonin gene polymorphisms: exploring the interplay of genes, brain, and behavior. Cognitive, Affective, \& Behavioral Neuroscience, 6(1), 44-52.

Classen, J., Gerloff, C., Honda, M., \& Hallett, M. (1998). Integrative visuomotor behavior is associated with interregionally coherent 
oscillations in the human brain. Journal of neurophysiology, 79(3), 1567-1573.

Day, D. V. (2000). Leadership development:: A review in context. The leadership quarterly, 11(4), 581-613.

Day, D. V., Gronn, P., \& Salas, E. (2004). Leadership capacity in teams. The leadership quarterly, 15(6), 857-880.

Finkelstein, S., Hambrick, D., \& Cannella, A. A. (1996). Strategic leadership. St. Paul: West Educational Publishing.

Gilgenkrantz, S. (1997). Le bonheur en héritage.

Hariri, A. R., \& Brown, S. M. (2006). Serotonin. American Journal of Psychiatry, 163(1), 12-12.

Harari-Dahan, O., \& Bernstein, A. (2014). A general approach-avoidance hypothesis of oxytocin: accounting for social and non-social effects of oxytocin. Neuroscience \& Biobehavioral Reviews, 47, 506-519.

Heisel, A. D., \& Beatty, M. J. (2006). Are cognitive representations of friends' request refusals implemented in the orbitofrontal and dorsolateral prefrontal cortices? A cognitive neuroscience approach to 'theory of mind'in relationships. Journal of Social and Personal Relationships, 23(2), 249-265.

House, R. J., \& Howell, J. M. (1992). Personality and charismatic leadership. The Leadership Quarterly, 3(2), 81-108.

Loftus, P. (1995). The pygmalion effect. Industrial and Commercial Training, 27(4), 17-20.

Mintzberg, H. M. (1976). Planning on the left side and managing on the right. Harvard Business Review, July-August, 4-6. 
Muller, M. N., \& Wrangham, R. W. (2004). Dominance, cortisol and stress in wild chimpanzees (Pan troglodytes schweinfurthii). Behavioral Ecology and Sociobiology, 55(4), 332-340.

Nolan, S. (2013). Effective Multi-unit Leadership. Local Leadership in Multisite Situations. Strategic HR Review.

Ochsner, K. N., \& Lieberman, M. D. (2001). The emergence of social cognitive neuroscience. American Psychologist, 56(9), 717.

Rosmalen, J. G. M. (2008). Stress, cortisol and the development of depression. Journal of Affective Disorders, (107), 38-39.

Waldman, D. A., Balthazard, P. A., \& Peterson, S. J. (2011). Leadership and neuroscience: Can we revolutionize the way that inspirational leaders are identified and developed?. Academy of Management Perspectives, 25(1), 60-74.

Wise, R. A. (2004). Dopamine, learning and motivation. Nature reviews neuroscience, 5(6), 483.

Zhe, J. W. X., \& Yazdanifard, R. (2015). The neuroscience of effective leadership; cultivation of a healthy corporate culture through neurochemicals. International Journal of Management, Accounting and Economics, 2(6), 584-594. 